\title{
Fortificación de harina de trigo con ácido fólico y hierro en Uruguay; implicancias en la nutrición
}

\author{
Fortification of wheat flour with \\ folic acid and iron in Uruguay; \\ implications for nutrition
}

\begin{abstract}
This study assessed folic acid (FA) and iron (Fe) content in flour fortified according to the levels regulated by law, and estimate the degree of adequacy of FA and Fe contained in French bread with the recommended dietary allowances (RDAs) and tolerable upper limits for different groups of Uruguayan population. Ten samples of flour and bread were analyzed. The median value for added FA and of the total Fe in flour was 2.3 and $48 \mathrm{mg} / \mathrm{kg}$ respectively. The recommended intake of bread covers the median value between $27 \%$ and $90 \%$ of the RDAs for Fe and between $69 \%$ and $104 \%$ of dietary folate equivalents in different groups. To sum up, fortification levels in flour adjusted to, exceeded or did not reach the regulated levels. Bread was a basic means to help cover RDAs in vulnerable people.

Key words: micronutrients; bread; iron; folates; nutritional recommendations; nutritional adequacy.
\end{abstract}

\section{INTRODUCCIÓN}

La fortificación de la harina de trigo se estableció como obligatoria en Uruguay a partir de un decreto ley del año 2006 y consiste en la adición de 2,4 mg de ácido fólico y $30 \mathrm{mg}$ de hierro (sulfato ferroso deshidratado o fumarato ferroso) por $\mathrm{kg}$ de harina blanca (1). Tiene como objetivos aumentar la ingesta de folatos, especialmente en mujeres en edad fértil para reducir la prevalencia de los defectos del tubo neural del recién nacido (2-5) y prevenir anemias de origen dietético, fundamentalmente en los grupos de mayor riesgo, es decir, niños, adolescentes, embarazadas y mujeres en edad reproductiva (6).

La harina de trigo fue seleccionada como vehículo de fortificación para estos nutrientes porque el pan es un alimento de consumo diario en Uruguay (7) y la adición, en la cantidad legislada y con una adecuada selección del compuesto químico, es posible sin alterar sus características organolépticas (8).

El nivel de fortificación en ácido fólico (AF) y hierro (Fe) es un factor determinante del aporte dietario de estos
Mónica Russo (1)

Marta Elichalt (1)

Daniel Vázquez (2)

Gabriela Suburú (3)

HugoTihista (4)

Marcela Godiño (5)

(1) Escuela de Nutrición y Dietética, Universidad de la República Oriental del Uruguay, Departamento de Alimentos, Uruguay. (2) Instituto Nacional de Investigación Agropecuaria, la Estanzuela, Uruguay. (3) Laboratorio Tecnológico del Uruguay, Departamento de Cereales, Oleaginosas, Productos Derivados. (4) Centros de Industriales Panaderos del Uruguay, Uruguay. (5) Mesa Nacional del Trigo, Uruguay.

Dirigir la correspondencia a: Profesora Mónica Russo Escuela de Nutrición y Dietética Departamento de Alimentos Universidad de la República Orienta Uruguay

E-mail: monrusso@gmail.com

Este trabajo fue recibido el 19 de Marzo de 2014 y aceptado para ser publicado el 16 de septiembre de 2014.

nutrientes (9). Niveles de fortificación superiores a lo establecido, especialmente cuando se asocian a un alto consumo de alimentos enriquecidos con AF y Fe y al uso de suplementos nutricionales, podrían determinar una mayor probabilidad de superar las ingestas máximas tolerables $(10,11)$. Esto cobra relevancia ya que estudios recientes muestran que ingestas elevadas de folatos podrían relacionarse con algún tipo de cáncer $(12,13)$ y con alteraciones neurológicas en el anciano $(14,15)$. Por otra parte, el exceso de Fe agrava el estrés oxidativo y acelera la degeneración tisular, por lo que se define como un factor de riesgo de ateroesclerosis y enfermedades cardiovasculares $(16,17)$.

La concentración de AF y Fe en la harina fortificada está determinada por los procesos utilizados a nivel de los molinos para la fortificación de harina.

Castillo et al. (18) en Chile encontraron variaciones en el contenido de AF en harinas fortificadas. En los resultados obtenidos, la mediana del contenido de AF en harinas eva- 
luada en tres años consecutivos fue de 1,1; 1,9 y $1,5 \mathrm{mg} / \mathrm{kg}$ respectivamente. Estos valores corresponden a 50; 13,6 y $31 \%$ menos que lo establecido en la norma chilena. Alrededor del $30 \%$ tenía contenidos superiores al 2,5 mg/ $\mathrm{kg}$ (13\% más que lo legislado). Además, no se detectó AF en hasta $20 \%$ de las muestras. Por tanto, es de interés conocer en qué medida la harina producida en Uruguay cumple con la legislación en los niveles de fortificación en AF y Fe.

Factores como los niveles de fortificación de la harina, las modificaciones por cocción del AF (19), así como la formulación realizada en la elaboración del pan son variables en la concentración de AF y Fe en este alimento.

Según Castillo et al (18), en estudios realizados en Chile se han hallado diferencias en la ingesta de AF proveniente del consumo aparente de pan, aplicando los diferentes niveles de fortificación encontrados en la harina. Los panes elaborados con las concentraciones de AF sobre el percentil 95 superaron al límite máximo tolerable con un consumo diario de dos a tres panes.

En Uruguay no se dispone de datos respecto al contenido de AF y Fe en los panes de panaderías (20). Por lo tanto, no se conoce en qué medida las cantidades de AF y Fe vehiculizadas en las porciones de pan incluidas en guías de consumo de alimentos recomendadas por el Ministerio de Salud Pública (MSP) constituyen un factor favorable o de riesgo según las recomendaciones nutricionales para diferentes grupos etarios. Estas guías establecen los diferentes grupos de alimentos y cantidades diarias a incluir para alcanzar las metas nutricionales establecidas para la población uruguaya (21).

Los objetivos de este estudio fueron evaluar el contenido de AF y Fe en harinas fortificadas con los niveles de adición legislados y estimar en pan francés elaborado en panaderías de Montevideo y Canelones el grado de adecuación de estos nutrientes con las ingestas diarias recomendadas (RDAs) y los límites máximos tolerables (ULs) para distintos grupos de la población uruguaya.

\section{MATERIALES Y MÉTODOS}

Muestras y método de recolección de datos

El estudio se llevó a cabo analizando diez muestras de harinas fortificadas provenientes de cinco molinos (dos lotes distintos por molino) y diez muestras de panes franceses elaborados en diez panaderías diferentes. Se seleccionaron molinos con diferente capacidad de producción y que en su conjunto abastecieran al 75\% del mercado interno del país.

Las muestras de harina se extrajeron en las panaderías seleccionadas a partir de dos lotes diferentes. Se retiraron $1 \mathrm{~kg}$ de la parte superior, del medio y de la parte inferior de una bolsa de harina en cada lote. Las muestras obtenidas se acondicionaron inmediatamente en envase hermético y se homogenizaron previo a realizar la determinación analítica de AF.

El pan se obtuvo de panaderías seleccionadas en conveniencia con el Centro de Industriales Panaderos del Uruguay (CIPU) entre los asociados de Montevideo y Canelones. Estas panaderías fueron abastecidas con harina de los lotes analizados.

El pan francés se elaboró a partir de la harina proveniente de la misma bolsa que se utilizó para generar las muestras de harina, siguiendo la formulación y el proceso de elaboración habitual de las panaderías. Inmediatamente a su cocción se retiraron tres panes de la bandeja superior, tres del medio y tres de la parte inferior del horno; se acondicionaron en bolsas herméticas y se congelaron a $-18^{\circ} \mathrm{C}$. Las muestras colectadas se homogenizaron previamente a su determinación.
Determinación de la cantidad de AF y Fe en harina fortificada y pan francés

La determinación de AF en harina y pan se realizó por método de cromatografía líquida (22). Esta técnica cuantifica únicamente el AF adicionado, no detecta el AF propio del alimento.

Para la determinación de Fe en harina de trigo, se utilizó el método de referencia que especifica el Reglamento Bromatológico Nacional (RBN) como exclusivo para harinas: método espectrofotométrico, en el cual el Fe de la harina reaccionó con la ortofenantrolina y se midió en el espectrofotómetro a 510 nm (23). En el pan, la medición se realizó por espectrometría atómica en muestras digeridas en sistema cerrado a alta presión por uso de microondas aplicando el método normalizado AOAC 999.10:2005 (24).

\section{Análisis de datos}

Para evaluar el contenido de AF y Fe en las harinas respecto a los niveles de fortificación legislados, se comparó lo establecido en el RBN (25) con la mediana del contenido de AF adicionado y Fe total de la harina analizada.

Con el fin de estimar el grado de adecuación de AF adicionado y Fe total contenidos en pan francés elaborado en panaderías con las RDAs y los ULs para distintos grupos de la población uruguaya, se tuvieron en cuenta los grupos de mayor riesgo (preescolar, mujer en edad fértil y embrazada) y además los adultos mayores.

A los efectos del cálculo se consideraron la mediana del contenido de AF adicionado y Fe total y los valores mínimos y máximos obtenidos en los panes analizados. Asimismo, se utilizaron $50 \mathrm{~g}$ de pan en niños (para una dieta de $1500 \mathrm{Kcal}$ ) y $150 \mathrm{~g}$ en mujeres en edad reproductiva, adultos mayores y embarazadas (para una dieta de $2200 \mathrm{Kcal}$ ), utilizando como referencia las guías de consumo de alimentos definidas por el MSP para cubrir las necesidades nutricionales de los principales grupos de la población (21). Como recomendaciones nutricionales se consideraron las RDAs y los ULs para AF y Fe (de 18\% de biodisponibilidad), establecidas por el Comité de Nutrición del Instituto de Medicina de los Estados Unidos $(10,11)$. Dado que las RDAs de AF se expresan como equivalentes dietarios de folatos (EDF) y la determinación en pan cuantifica el AF adicionado, este fue convertido en EDF utilizando un factor de conversión 1,67 (10). El grado de adecuación se expresó como el porcentaje de las RDAs que fueron cubiertas con las concentraciones de AF y Fe encontradas en el pan según las porciones establecidas para los diferentes grupos estudiados.

\section{RESULTADOS Y DISCUSIÓN}

En la tabla 1 se observa que la mediana del contenido de AF adicionado en harina fue muy próxima al valor legislado $(2,4 \mathrm{mg} / \mathrm{kg})$. Sin embargo, en más de la mitad de los resultados los valores estuvieron por debajo de lo legislado, $(37,5 \%)$ menos. Por otra parte, el valor máximo analizado casi duplicó lo establecido para la fortificación de harina. En el caso de $\mathrm{Fe}$, la diferencia entre el valor mínimo y el máximo obtenido alcanzó más del doble, pero, a diferencia del AF, todos los valores superaron lo legislado. Esto puede explicarse por la metodología utilizada para la determinación analítica de Fe que cuantifica el total contenido en la harina (el natural del alimento y el agregado para su fortificación), a diferencia de la utilizada para la cuantificación de AF que sólo determina el adicionado. Al sustraer de los valores obtenidos el contenido natural de Fe de la harina (11 a $12 \mathrm{mg} / \mathrm{kg}$ ) declarado en la Tabla Uruguaya de Composición Química (20), la mediana 
del Fe adicionado superó el valor legislado (36 $\mathrm{mg} / \mathrm{kg}$ ) y en una de la muestras el contenido fue algo inferior ( $7 \%$ menos).

En estudios previos $(26,18)$ también se reportaron valores por encima y por debajo de lo legislado. Castillo et al. (18) sugieren que la dispersión entre los valores hallados puede generarse por una granulometría inadecuada de la mezcla de la vitamina y el mineral que limita la homogenización, por mezclado inadecuado o por tiempo insuficiente. En tanto que los niveles superiores también pueden corresponder a una sobredosificación para asegurar el cumplimiento de los niveles establecidos.

En pan francés también se observó variabilidad en los valores hallados, con menor diferencia entre los contenidos mínimos y máximos de AF y Fe en comparación con la harina (AF 2 vs 3 y Fe 1,5 vs 2 veces). Esto puede deberse a que el proceso de elaboración del pan requiere de un amasado intenso y prolongado que favorece la homogenización de los constituyentes de la harina utilizada para su elaboración. En todos los casos los valores mínimos, máximos y de las medianas fueron, como es de esperar, menores a los obtenidos en la harina fortificada, porque en la elaboración de la masa se adiciona agua, lo cual aumenta la proporción de humedad en el producto terminado y consecuentemente disminuye la concentración de los nutrientes.

En la tabla 2 se observa que la mediana de la ingesta de AF adicionado expresada como EDF, proveniente del pan, alcanzó a cubrir al menos los 2/3 de las RDAs en todos los grupos estudiados y las superaron ligeramente en los grupos de mujeres en edad reproductiva y adultos mayores (104\%). Al considerar el contenido máximo de AF adicionado en pan, la ingesta de $150 \mathrm{~g}$ superó las RDAs en 1,5 veces para estos grupos de la población. Por tanto, la ingesta de AF adicionado en mujeres en edad fértil y adultos mayores, estimada a través del consumo de pan, podría determinar la existencia de grupos de la población con mayor probabilidad de ingestas excesivas de este nutriente. No obstante, en ninguno de los casos la ingesta de AF alcanzó el UL.

Sin embargo, investigaciones previas en Chile, que han estudiado la ingesta de AF en el periodo post-fortificación en función del consumo aparente de pan, han informado que los adultos (mujeres y hombres) tenían ingestas superiores al UL en los grupos con mayores ingestas diarias de pan (18).

Considerando que las estimaciones de AF realizadas en este estudio se basaron exclusivamente en la cantidad adicionada y en una cantidad de pan estimada tomando como referencia las guías de consumo de alimentos definidas por el MSP y que en estudios nacionales se citan grupos de la población que la superan (180 g/día), pueden esperarse ingestas diarias de este nutriente superiores a lo estimado, especialmente cuando se asocian a un consumo frecuente de otros productos elaborados con harina de trigo fortificada (7).

Por otra parte, se ha demostrado que altas dosis de AF asociadas al déficit de vitamina B12 aumentan el riesgo vinculado al deterioro cognitivo y neurológico en adultos mayores y el riesgo de desarrollar cáncer de colon en individuos con lesiones neoplásicas incipientes (27). A su vez, se ha señalado que ingestas altas de AF son beneficiosas para prevenir cáncer de colon en portadores sanos y en consumidores de alcohol $(28,29)$.

Los datos de este estudio muestran que el contenido

\section{TABLA 1}

Contenido de ácido fólico adicionado y hierro total en harina de trigo fortificada y pan francés elaborado en panaderías.

\begin{tabular}{|c|c|c|c|c|c|c|}
\hline \multirow[b]{2}{*}{$\begin{array}{l}\text { Concentración } \\
(\mathrm{mg} / \mathrm{kg})\end{array}$} & \multicolumn{3}{|c|}{$\begin{array}{l}\text { Harina de trigo fortificada } \\
\qquad(n=10)\end{array}$} & \multicolumn{3}{|c|}{$\begin{array}{l}\text { Pan francés } \\
\qquad(n=10)\end{array}$} \\
\hline & Mínimo & Mediana & Máximo & Mínimo & Mediana & Máximo \\
\hline Ácido fólico & 1,5 & 2,3 & 4,7 & 1,1 & 1,7 & 2,3 \\
\hline Hierro & 40 & 48 & 75 & 32 & 39 & 48 \\
\hline
\end{tabular}

\section{TABLA 2}

Estimación de la ingesta de ácido fólico adicionado (expresado en Equivalentes Dietarios de Folatos) en la cantidad diaria recomendada de pan, según distintos niveles de concentración, en diferentes grupos de la población.

\begin{tabular}{|c|c|c|c|c|}
\hline & \multirow[t]{2}{*}{$\operatorname{Pan}^{a}$ (g/día) } & \multicolumn{3}{|c|}{ Ingesta EDF ${ }^{b}(\mu g / d i ́ a)$} \\
\hline & & Mínimo & Mediana & Máximo \\
\hline Preescolar & 50 & $92(46)$ & $148(74)$ & $192(96)$ \\
\hline Mujer en edad fértil & 150 & $276(69)$ & $414(104)$ & $576(144)$ \\
\hline Adulto mayor & 150 & $276(69)$ & $414(104)$ & $576(144)$ \\
\hline Embarazada & 150 & $276(46)$ & $414(69)$ & $576(96)$ \\
\hline $\begin{array}{l}\text { Ingesta diaria recomendada } \\
\text { Equivalentes Dietarios de Fol } \\
\text { Los valores entre paréntesis ex } \\
\text { Unidos (10) que fueron cubie } \\
\text { alimentos para distintos grup }\end{array}$ & $\begin{array}{l}\text { en las Guías Alin } \\
\text { es de las ingestas } \\
\text { ades de ácido fól } \\
\text { (21). }\end{array}$ & $\begin{array}{l}\text { alimentos por } \\
\text { de EDF por } \\
\text { n según las po }\end{array}$ & $\begin{array}{l}\text { de Salud Públ } \\
\text { Nutrición del I } \\
\text { blecidas en las }\end{array}$ & $\begin{array}{l}\text { Medicina de los Estados } \\
\text { entarias Basadas en }\end{array}$ \\
\hline
\end{tabular}


de AF en los panes estudiados puede ser beneficioso para alcanzar a cubrir las necesidades diarias de este nutriente en algunos grupos de la población, mientras que pueden no ser adecuados para algunas situaciones específicas.

Respecto a la ingesta de hierro proveniente del pan, en los grupos de niños y embarazadas la mediana del contenido de Fe en pan cubrió una cuarta parte de las necesidades diarias de este mineral y alcanzó hasta un $42 \%$ en el caso de concentraciones máximas (tabla 3 ). En las mujeres en edad fértil el consumo establecido de pan para una alimentación saludable aportó entre uno a dos tercios de las necesidades diarias de Fe, según los valores mínimos y máximos encontrados. Por otro lado, en los adultos mayores la mediana cubrió casi la totalidad de las RDAs, y las superó cuando se estimó la ingesta con el valor máximo encontrado.

Entre los grupos de mayor riesgo a desarrollar anemias por déficit alimentario de $\mathrm{Fe}$, es decir, niños, mujeres en edad fértil y embarazadas (30), los valores hallados cobran relevancia al considerar lo que reflejan estudios nacionales (7) respecto a la baja ingesta de alimentos ricos en Fe hemínico (carnes, pescado e incluso aves) en la población de menores recursos. La inclusión diaria de pan, según los resultados obtenidos en este estudio, puede ser una importante contribución para cubrir las necesidades de Fe. Como se mencionó, el pan es un alimento que se incluye diariamente en la alimentación de los uruguayos, además de ser de fácil acceso y bajo costo (7). Sin embargo, para prevenir deficiencias de Fe de origen alimentario, además del pan con harina fortificada, se requiere incluir en la alimentación diaria otros alimentos fuentes de Fe o que estén fortificados con este mineral.

En conclusión, los resultados de este estudio muestran que si bien en todas las harinas evaluadas se ha realizado la fortificación en AF y Fe, se observaron tanto valores que adecuaron como otros que superaron o no alcanzaron lo establecido en la legislación. Respecto al grado de adecuación entre la ingesta de AF y Fe proveniente de la cantidad diaria estimada de pan en base a las guías de consumo de alimentos definidas por el MSP y las recomendaciones nutricionales de estos nutrientes, las muestras analizadas fueron una contribución nutricionalmente significativa en la alimentación de los grupos vulnerables, para los cuales la fortificación constituyó un objetivo. Sin embargo, en los adultos mayores, exclusivamente la ingesta de pan alcanzó a cubrir las RDAs de AF y $\mathrm{Fe}$, aunque no alcanzó los ULs de estos nutrientes. Estos son aspectos a considerar para optimizar los beneficios de la fortifi- cación en AF y Fe minimizando los riesgos de sobreexposición innecesaria en algunos grupos.

Dentro de las limitaciones de este estudio se reconoce el restringido tamaño de la muestra, el cual no permite extrapolar los resultados al universo de las panaderías del país. Existe por lo tanto la necesidad de ampliar el estudio a modo de medir el nivel de cumplimiento de la fortificación de la harina de trigo, así como de conocer la contribución nutricional que tiene el pan francés al cumplimiento de las necesidades de Fe y AF particularmente en los grupos de mayor vulnerabilidad.

\section{RESUMEN}

La presente investigación evaluó el contenido de ácido fólico (AF) y hierro (Fe) en harinas fortificadas y comparar con los niveles de adición legislados, estimando el grado de adecuación de AF y Fe contenidos en pan francés con las ingestas diarias recomendadas (RDAs) y los límites máximos tolerables para distintos grupos de la población uruguaya. Se analizaron diez muestras de harina y de pan. El valor de la mediana de AF adicionado y del Fe total, en harinas fue 2.3 y $48 \mathrm{mg} / \mathrm{kg}$ respectivamente. La ingesta recomendada de pan cubre en el valor de la mediana entre un $27 \%$ y $90 \%$ de las RDAs de Fe y entre el $69 \%$ y $104 \%$ de Equivalentes Dietarios de Folatos en diferentes grupos. En conclusión, los niveles de fortificación en harina adecuaron, superaron o no alcanzaron lo legislado. El pan constituyó un vehículo básico para cubrir las RDAs en la población vulnerable.

Palabras clave: micronutrientes; pan; hierro; folatos; recomendaciones nutricionales; adecuación nutricional.

\section{BIBLIOGRAFÍA}

1. Ministerio de Salud Pública. Norma técnica para la fortificación de harinas en Uruguay; decreto No. 130/006. Disponible en: http://www.msp.gub.uy/marco-normativo/ decreto-130006-sobre-fortificaci\%C3\%B3n-de-harinas. =[consulta 15 septiembre 2012]. 2006.

2. Smithells RW, Sheppard S, Schorah CJ, Seller MJ, Nevin $N C$, Harris R. Apparent prevention of neural tube defects by periconceptional vitamin supplementation, Arch Dis Child 1981; 56:911-8.

3. Werler MM, Shapiro S, Mitchell AA. Periconceptional folic acid exposure and risk of occurrent neural tube defects, JAMA 1993; 269:1257-61.

4. Shaw GM, Schaffer D, Velie EM, Morland $K$, Harris JA. Periconceptional vitamin use dietary folate, and the oc-

TABLA 3

Estimación de la ingesta de hierro en la cantidad diaria recomendada de pan, según distintos niveles de concentración, en diferentes grupos de la población.

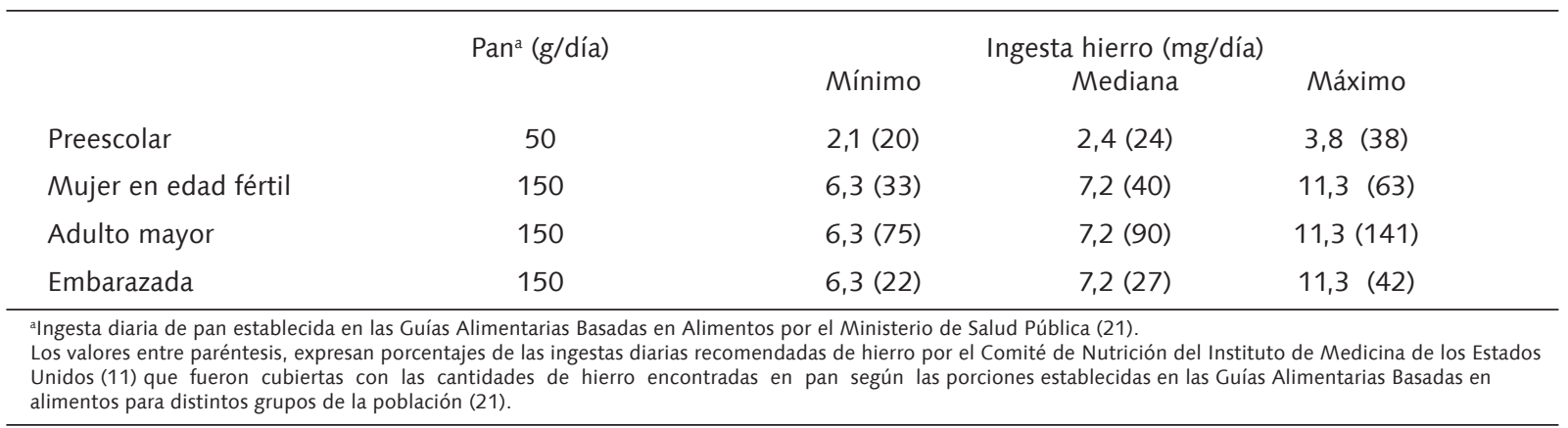


currence of neural tube defects, Epidemiology 1995; 6:219-26.

5. Hursthouse NA, Gray AR, Miller JC, Meredith RC, Houghton $L A$. Folate status of reproductive age women and neural tube defect risk: the effect of long-term folic acid supplementation at doses of 140 and $400 \mathrm{mcg}$ per day, Nutrients 2011; 3:49-62.

6. Zabala R, Waisman I, Corelli M, Tobler B. Ácido fólico para prevenir defectos del tubo neural: consumo e información en mujeres en edad fértil de la Región Centro Cuyo, Arch Argent Pediatr. 2008; 106:295-301.

7. Bove I, Cerruti I. Los alimentos y las bebidas en los hogares: ¿un factor de protección o de riesgo para la salud y el bienestar de los uruguayos? Encuesta Nacional de Gastos e Ingresos de los Hogares 2005-2006. INE, Montevideo, 2008. Disponible en: http://www.ine.gub.uy/ biblioteca/engih2006/Los\%20alimentos\%20y\%20las\%20 bebidas\%20en \%20los\%20hogares\%20(versi\%F3n\%20 final).pdf $=$ [consulta 20 agosto 2012]

8. Boccio J, Bressan J. Fortificación de alimentos con hierro y zinc: pros y contras desde el punto de vista alimenticio y nutricional, Rev Nutr. 2004; 17:71-8.

9. Rader JI. Folic acid fortification, folate status and plasma homocysteine, J Nutr. 2002; 132 (8 Suppl):2466S-70S.

10. Institute of Medicine, Food and Nutrition Board. National Academies. Dietary Reference Intakes for Thiamin, Riboflavin, Niacin, Vitamin B6, Folate, Vitamin B12, Panthotenic Acid, Biotin and Choline, 1998, p.196-395. Disponible en: http://www.nap.edu/openbook.php?record $i d=6015 \&$ page $=196$. $=$ [consulta 12 junio 2012].

11. Institute of Medicine, Food and Nutrition Board. National Academies. Dietary Reference Intakes for Vitamin A, Vitamin K, Arsenic, Boron, Chromium, Copper, Iodine, Iron, Manganese, Molybdenum, Nickel, Silicon, Vanadium and Zinc, 2001, p. 290-393. Disponible en: http://www.nap. edu/openbook.php?record_id=10026\&page $=290 .=[\mathrm{con}-$ sulta12 junio 2012].

12. Mattias J, Paul N, Naomi E. Circulating and concentration of folate and vitamin $B 12$ concentration in relation to prostate cancer risk: results from the European Prospective Investigation into Cancer and Nutrition study, Cancer Epidemiol Biomarkers Prev. 2008; 172:79-285.

13. Castillo C, Tur J, Uauy R. Suplementación con ácido fólico y prevención de recurrencias de adenomas colorrectales; revisión sistemática, Nutr Hosp. 2012; 27:13-21.

14. Miller J, Garrod M, Allen L, Haan M, Green R. Metabolic evidence of vitamin B12 deficiency, including high homocysteine and methylmalonic acid and low holotranscobalamin, is more pronounced in older adults with elevated plasma folate, Am J Clin Nutr. 2009; 90:1586-92.

15. Selhub J, Morris $M$, Jacques $P$, Rosenberg I. Folate-vitamin
B12 interaction in relation to cognitive impairment, anemia, and biochemical indicators of vitamin B12 deficiency, Am J Clin Nutr. 2009; 89:707S-11S.

16. Edison ES, Bajel A, Chandy M. Iron homeostasis: new players, newer insight, Eur J Haematol. 2008; 81:411-424.

17. Toxqui L, De Piero A, Courtois V, Bastida S, Sanchez-Muniz FJ, Vaquero PM. Deficicienca y sobrecarga de hierro; implicaciones en el estrés oxidativo y la salud cardiovascular, Nutr Hosp. 2010; 25:350-65.

18. Castillo C, Tur J, Uauy R. Fortificación de la harina de trigo en ácido fólico en Chile. Consecuencias no intencionadas, Rev Med Chil. 2010; 138:832-40.

19. Fennema O. Química de los alimentos. Acribia 3a ed., Zaragoza, 2010.

20. Tor E, Herrera MA. Tabla de composición de alimentos de Uruguay. Instituto Nacional de Alimentación, Montevideo, 2002.

21. Ministerio de Salud Pública. Dirección General de la Salud. Programa Nacional de Nutrición. Manual para la promoción de prácticas saludables en la población uruguaya. Montevideo, 2005, p. 20.

22. Konings $E$. A validated liquid chromatographic method for determining folates in vegetables, milk powder, liver and flour, J AOAC 1999; 82 N¹:119-27.

23. American Association of Cereal Chemists International (AACC International). Approved methods of analysis international, $11^{\mathrm{a}} \mathrm{ed}$. Washington D.C., 2010.

24. Association of Official Analytical Chemists (AOAC). Methods of analysis of AOAC International, $18^{\mathrm{a}} \mathrm{ed}$. Washington D.C., 2007.

25. Ministerio de Salud Pública. Reglamento Bromatológico Nacional (Uruguay). Decreto No. $315 / 994$ de fecha 05/07/1994. $3^{a}$ ed.Montevideo, IMPO, 2008, p. 460.

26. Lakshmi K. Folic acid, total folate and food folate in pizza and other fast food. Master of Science. Bangalore University, India, 2005.

27. Cole BF, Baron JA, Sandler RS, Haile RW, Ahnen DJ, Bresalier RS. Polyp Prevention Study Group. Folic acid for the prevention of colorectal adenomas: a randomized clinical trial. JAMA 2007; 297 (21):2351-9.

28. Bailey LB. Folate, methyl-related nutrients, alcohol, and the MTHFR 677C-T polymorphism affect cancer risk: intake recommendations. J Nutr. 2003; 133 (11 Suppl 1):3748S-53S.

29. Kim YI. 5, 10-Methylenetetrahydrofolate reductase polymorphisms and pharmacogenetics: A new role of single nucleotide polymorphisms in the folate metabolic pathway in human health and disease. Nutr Rev 2005; 63:398-407.

30. 30. Díaz MS, Guerra PH, Campos SM, Letelier MA, Olivares M. Prevalencia de hierro en preescolares de la comuna $L a$ Pintana Rev Chil Nutr. 2002; 29:1-13. 